\title{
Fast Optical Beamforming Architectures for Satellite-Based Applications
}

\author{
B. Vidal, ${ }^{1}$ T. Mengual, ${ }^{2}$ and J. Martí1 \\ ${ }^{1}$ Nanophotonics Technology Center, Universitat Politècnica de València, 46022 Valencia, Spain \\ ${ }^{2}$ DAS Photonics, Ciudad Politécnica de la Innovación, 46022 Valencia, Spain
}

Correspondence should be addressed to B. Vidal, bvidal@dcom.upv.es

Received 19 June 2012; Accepted 17 September 2012

Academic Editor: Nathan J. Gomes

Copyright ( 2012 B. Vidal et al. This is an open access article distributed under the Creative Commons Attribution License, which permits unrestricted use, distribution, and reproduction in any medium, provided the original work is properly cited.

\begin{abstract}
Photonic technology offers an alternative implementation for the control of phased array antennas providing large time bandwidth products and low weight, flexible feeding networks. Measurements of an optical beamforming network for phased array antennas with fast beam steering operation for space scenarios are presented. Experimental results demonstrate fast beam steering between 4 and $8 \mathrm{GHz}$ without beam squint.
\end{abstract}

\section{Introduction}

The evolution of satellite communication and Earth observation missions has shown a clear trend towards systems with higher performance resulting in higher complexity. More in particular, a key requirement for modern space missions is the operation at wide bandwidths. As far as communication satellites are concerned, wide bandwidths are of great interest in order to accommodate broadband data connections, multiuser operation rates, and wider communications coverage. On the other side, Earth observation platforms also benefit from broadband microwave instruments which can provide a larger number of channels from which more complete and diverse remote sensing information can be extracted.

Additionally, advanced satellite missions are expected to provide high versatility through capabilities such as scanning and multibeam operation which combined to onboard information processing that allow the assignment of resources dynamically.

Wide bandwidth operation requires antenna array systems capable of providing true-time delay (TTD) to avoid beam squint (i.e., changes in the beam steering angle with frequency). However microwave implementations of TTD beamforming networks are rather bulky and heavy with poor harness characteristics, sensitivity to electromagnetic interference and a relatively large crosstalk level which degrade the performance of remote sensing instruments.
On the other hand, photonics allows the implementation of TTD beamforming networks without the problems associated with microwave implementations. Optical beamforming has been studied since the early 90s [1] because it provides many advantages over microwave and digital beamforming, such as light-weight, small size, wideband operation, flexibility, remoting capability, and immunity to electromagnetic interference. Different optical beamforming networks have been presented using a variety of components and arrangements [1-9].

Additionally, some optical beamforming architectures [10-13] offer the capability to control a set of independent beams simultaneously, that is, multibeam operation, although at the cost of increasing the complexity.

This paper reports on the feasibility of implementing fast multibeam TTD optical beamforming architectures to be used in future wideband satellite missions.

\section{Fast Beam Steering Applications}

Among the many applications of communications and Earth observation satellites [14] several of them would benefit from the capability of steering broadband beams with high speed. Low Earth Orbit (LEO) satellites for remote sensing usually employ a store and forward approach to send data to networks of receiving ground stations, such as Synthetic 


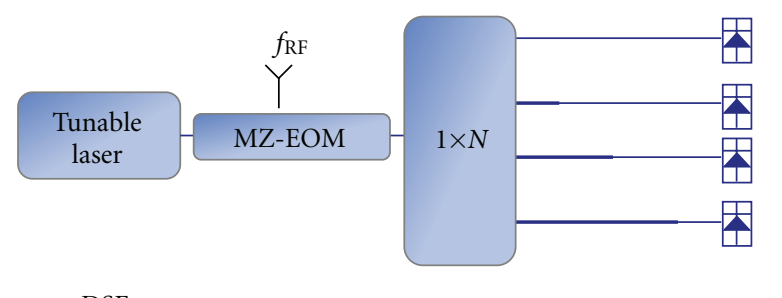

— DSF

- SMF

Figure 1: Optical beamformer based on a fast tunable laser.

Aperture Radar (SAR) instruments that require the use of several ground stations as well as large capacity onboard memory. Data transmission in this approach can be improved using broadband antenna systems with fast beam steering to track Earth stations and improve signal-to-noise ratio through antenna directivity at the receiver. Additionally, SAR systems show a clear trend toward broader bandwidths and Spot-SAR applications require both wide bandwidths and fast beam switching.

Future Medium Earth Orbit (MEO) constellations will require intersatellite broadband links to cover Internet traffic on satellite mobile phones, as the Iridium constellations actually do to provide voice calls and very limited data traffic. Fast beam steering networks with a limited number of simultaneous beams to track a set of moving satellites will be needed for the deployment of this kind of service.

Finally, satellites at the Geostationary Orbit (GEO) providing Very Small Aperture Terminal (VSAT) applications will apply frequency reuse strategies through fast hopping beam systems.

These and other satellite applications would benefit from the flexibility that fast beam steering broadband satellites could provide.

\section{Optical Beamforming Architectures}

The wide range of optical beamforming architectures proposed so far tackles different issues and are matched for specific scenarios. The definition of the scenario highly determines the most suitable beamforming architecture. In particular, in this Letter, fast beam steering and multibeam operation with a small/moderate number of antenna elements were identified as the key points for the applications identified in Section 2.

Architectures capable of fulfilling previous requirements include beamforming networks based on a fast tunable laser and a dispersive prism [12]. As shown in Figure 1, the output of the laser is amplitude modulated and launched to a dispersive prism made of a combination of highly dispersive fiber or standard single mode fiber and dispersion shifted fiber. By tuning the wavelength of the optical carrier a different time delay profile can be obtained due to the different time delay introduced by the fact that total dispersion in each branch of the prism is different. In this way, using a fast tunable laser, fast TTD beam steering can be implemented.

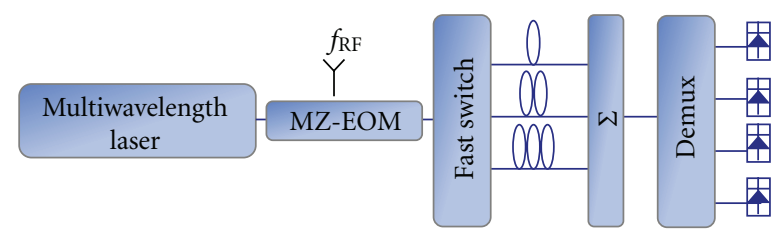

FIGURE 2: Optical beamformer based on a fast switch and dispersive fiber.

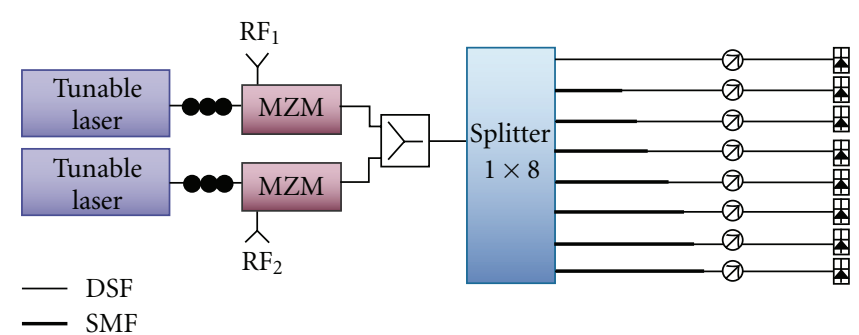

FIGURE 3: Block diagram of a fast multibeam forming network.

A second option is based on a fast switch (Figure 2) [2]. In this case, the architecture needs as many optical carriers as antenna elements (or subarrays if TTD is provided only to subarray level and phase control within the subarrays is done using microwave phase shifters) with a constant wavelength spacing between them. This set of optical carriers is amplitude modulated and using the fast switch is routed to a particular dispersive medium (e.g., a reel of a particular length). Dispersion introduces a relative time delay between optical carriers. Then the signals are combined and each optical carrier is demultiplexed to one photodiode. This architecture can be implemented using cascaded $2 \times 2$ fast optical switches or a $1 \times N$ optical switch. Since the cost of a fast optical switch can be significant, the solution based on a single switch should be preferred.

An additional important constraint is the need of multibeam operation. If multibeam is added to the requirement of fast beam steering, the architecture shown in Figure 1 is more suitable than the one of Figure 2. The OBFN based on a dispersive prism has straightforward multibeam capability, each additional beam just requires another optical source and external modulator. The TTD unit provides several time delay profiles when several optical carriers are used simultaneously. On the other hand, the architecture of Figure 2 requires the replication of the fast switch in order to provide multibeam operation (as many optical switches are needed as antenna beams).

\section{Experimental Results}

Figure 3 shows the block diagram of a fast beam steering architecture with two simultaneous beams.

Fast beam steering is obtained through a fast tunable laser (Intune Altonet 1200, central wavelength $1545 \mathrm{~nm}$ approximately) which provides a CW optical carrier whose wavelength can be rapidly tuned among a set of 84 channels with a spacing of $50 \mathrm{GHz}( \pm 2.5 \mathrm{GHz})$. The beamformer was 


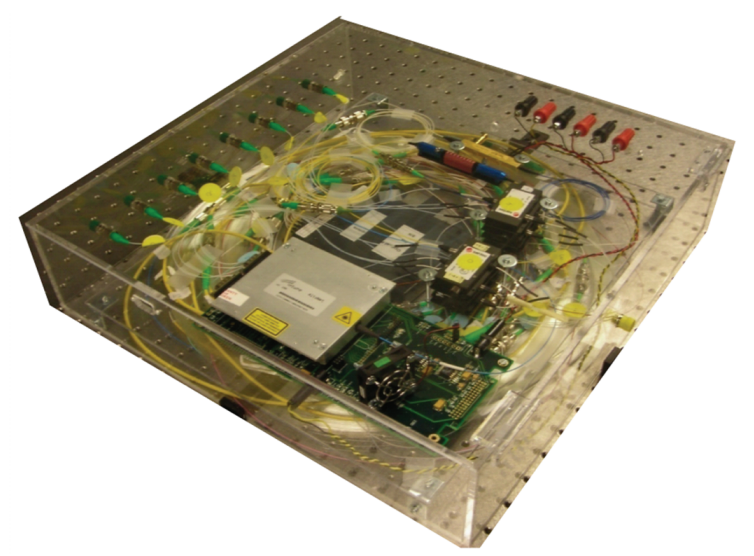

FIgURE 4: Picture of the beamformer based on a dispersive prism.

designed to steer the main lobe to broadside at the central wavelength of the optical source (1545 nm approximately). Moreover, to provide fully versatility the beamwidth of each beam could be controlled (i.e., the amplitude contribution of each beam in the antenna elements has to be controlled independently) by means of VOAs (variable optical attenuator). Figure 4 shows a picture of complete beamforming network.

Amplitude and time delay measurements of the eight channels of the beamformer were carried out to derive the radiation pattern for different beam steering angles (i.e., different laser wavelengths). From these measurements, the radiation pattern was derived assuming an antenna array whose elements were set at $0.7 \lambda$, where $\lambda=c /(8 \mathrm{GHz})$. The minimum steering step that the beamformer can achieve using the optical source of the setup is around $1^{\circ}$ and it can be swept from $46.9^{\circ}$ to $-41.9^{\circ}$. This particular resolution is only given by the optical source used in the experiments.

Figure 5, as an example, shows one of the measurements obtained. It presents the estimated radiation pattern for channel $32(1552.90 \mathrm{~nm})$. It can be seen that the main beam is steered at $20.8^{\circ}$ and that the beam steering angle is the same for all the frequencies (i.e., there is no beam squint due to the fact that beam steering is implemented using time delays instead of phase shifts). In addition to the fact that radiation pattern shows no beam squint, also the sidelobe levels are consistent with expected values for uniform amplitude distributions (i.e., around 13.2 dB for a large array). Figure 6 shows how the radiation pattern can be steered by changing the wavelength.

The proposed architecture allows the reconfiguration of the radiation pattern shape. It is shown in Figure 7 where a uniform, triangular, and Taylor amplitude distributions were performed. The radiation pattern reshaping applies to both beams since it is implemented using VOAs which affect equally both optical sources.

To measure the laser switching speed the setup of Figure 8 was implemented. The laser was configured to switch between two wavelengths and an optical filter was used to attenuate one of these wavelengths. In this way, an electrical signal with two levels can be measured at the

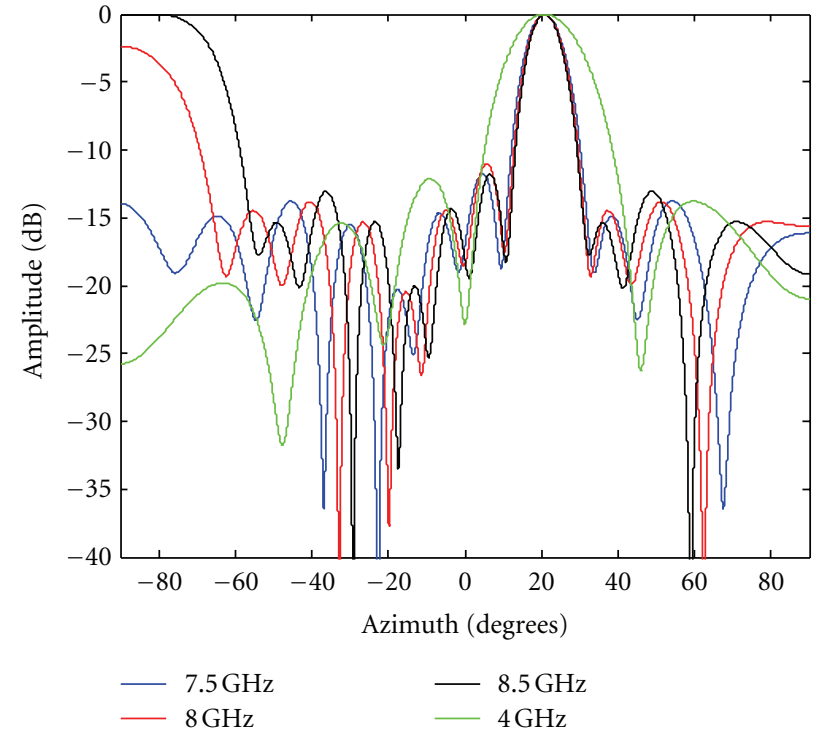

FIGURE 5: Radiation pattern of 8-element antenna calculated from the amplitude and phase when the laser is set at channel 32 $(1552.90 \mathrm{~nm})$.

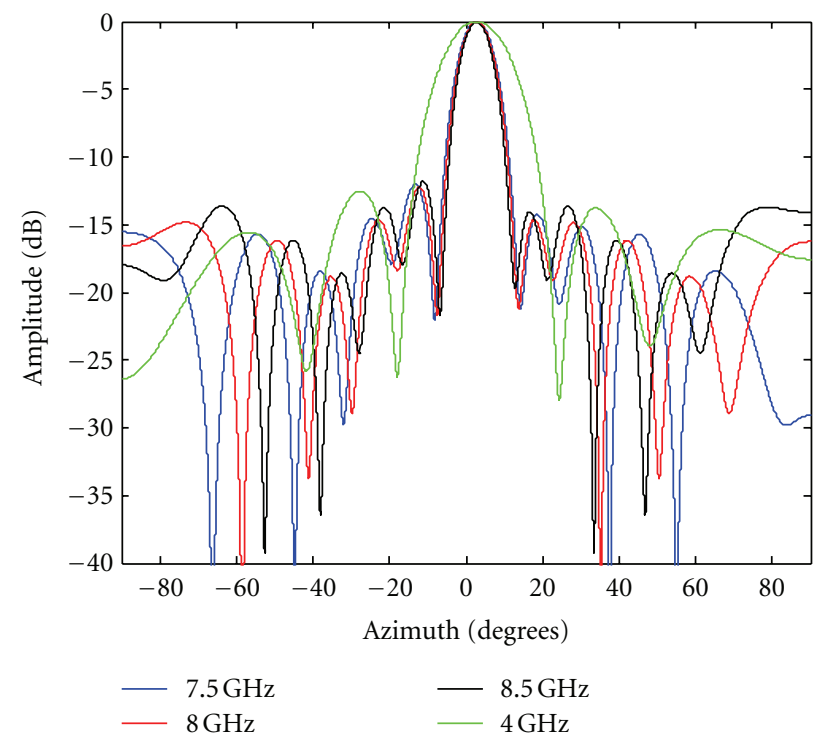

FIGURE 6: Radiation pattern of 8-element antenna calculated from the amplitude and phase when the laser is set at channel 50 (1545.69).

output of a photodiode using an oscilloscope and from these measurements derive the laser switching speed.

As shown in Figure 9, measurements were carried out using two channels of the optical source (in particular channels 37 and 50) with a dwell time in each state of $200 \mathrm{~ns}$ (this is the minimum dwell time allowed by the laser evaluation board software). From the results it can be concluded that the laser rise time was $700 \mathrm{ps}$, the fall time was $1.2 \mathrm{~ns}$, that is, a switching time of around $1 \mathrm{~ns}$. Finally, the measurements show that time in each state was around 440 ns. Since the 


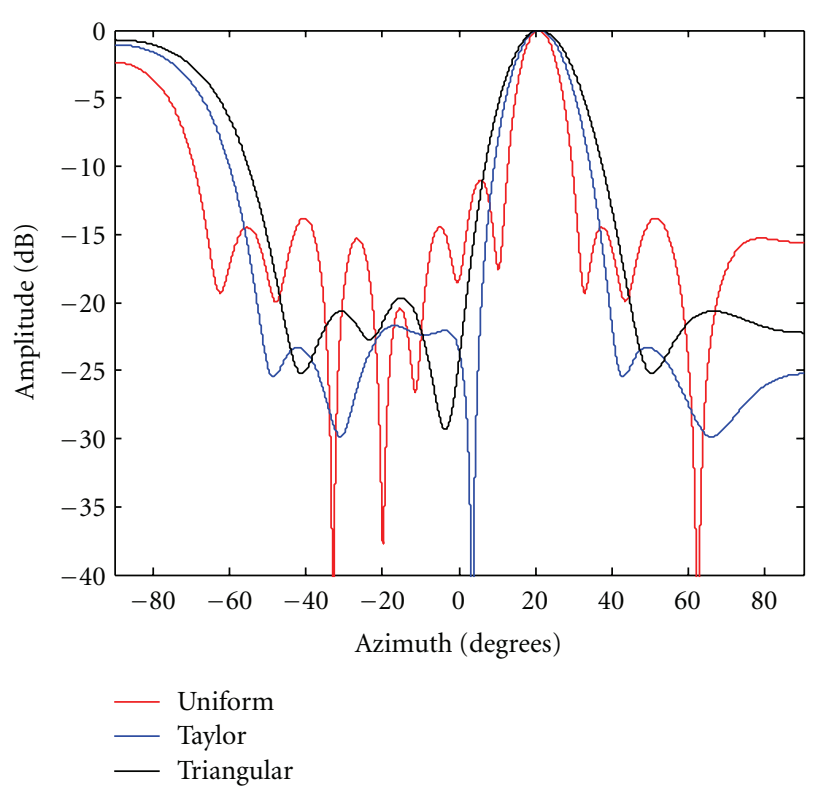

Figure 7: Radiation pattern for a uniform, Taylor and triangular amplitude distributions for steering at $20.8^{\circ}$ (channel 32, $1552.90 \mathrm{~nm}$ ).

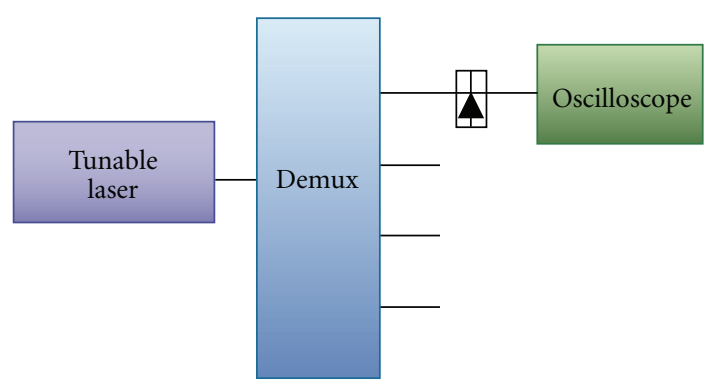

FIgURE 8: Setup for laser speed characterization.

optical source can change from any channel to any other, this architecture allows an arbitrary pattern of steering angles for beam hopping services. For continuous scans the time delay of the sweep would be given by the number of steps which is given by the number of channels of the optical source.

\section{Conclusion}

Fiber-optic signal processing provides interesting features to implement beamforming networks especially suitable for satellite applications. Key requirements in satellites include low weight, remote delivery to feed deployable array antennas, immunity to electromagnetic interference, and robustness to space radiation. These characteristics match appropriately with inherent features of fiber-optic systems. Additionally, broad bandwidth and fast operation obtained through photonic technology pave the way for the development of new satellite systems with advances capabilities and improved resilience. The use of optical beamforming architectures for satellite applications where fast beam steering

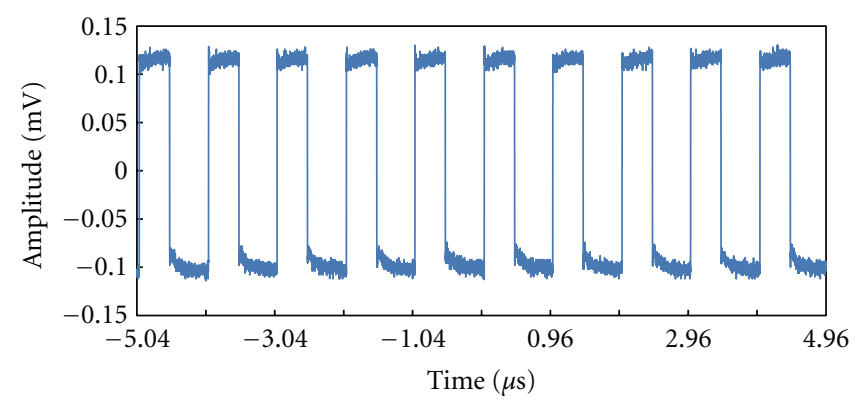

Figure 9: Electrical signal at the oscilloscope as shown in Figure 7 for a dwell time of $200 \mathrm{~ns}$.

is required has been reviewed. Measurements of an optical beamforming architecture showing fast operation (switching time of around $1 \mathrm{~ns}$ ) have been provided between 4 and $8 \mathrm{GHz}$ showing the feasibility of photonic fast beamforming.

\section{References}

[1] W. Ng, A. A. Walston, G. L. Tangonan, I. L. Newberg, J. J. Lee, and N. Bernstein, "The first demonstration of an optically steered microwave phased array antenna using true-timedelay," Journal of Lightwave Technology, vol. 9, no. 9, pp. 11241131, 1991.

[2] D. T. K. Tong and M. C. Wu, "A novel multiwavelength optically controlled phased array antenna with a programmable dispersion matrix," IEEE Photonics Technology Letters, vol. 8, no. 6, pp. 812-814, 1996.

[3] B. Vidal, J. L. Corral, M. A. Piqueras, and J. Martí, "Optical delay line based on arrayed waveguide gratings' spectral periodicity and dispersive media for antenna beamforming applications," IEEE Journal on Selected Topics in Quantum Electronics, vol. 8, no. 6, pp. 1202-1210, 2002.

[4] B. Vidal, J. L. Corral, and J. Martí, "Optical delay line employing an arrayed waveguide grating in fold-back configuration," IEEE Microwave and Wireless Components Letters, vol. 13, no. 6, pp. 238-240, 2003.

[5] B. Vidal, T. Mengual, C. Ibáñez-López et al., "Simplified WDM optical beamforming network for large antenna arrays," IEEE Photonics Technology Letters, vol. 18, no. 10, pp. 1200-1202, 2006.

[6] M. A. Piqueras, G. Grosskopf, B. Vidal et al., "Optically beamformed beam-switched adaptive antennas for fixed and mobile broad-band wireless access networks," IEEE Transactions on Microwave Theory and Techniques, vol. 54, no. 2, pp. 887-899, 2006.

[7] B. Vidal, T. Mengual, C. Ibáñez-López, and J. Martí, “Optical beamforming network based on fiber-optical delay lines and spatial light modulators for large antenna arrays," IEEE Photonics Technology Letters, vol. 18, no. 24, pp. 2590-2592, 2006.

[8] L. Jofre, C. Stoltidou, S. Blanch et al., "Optically beamformed wideband array performance," IEEE Transactions on Antennas and Propagation, vol. 56, no. 6, pp. 1594-1604, 2008.

[9] L. Zhuang, C. G. H. Roeloffzen, A. Meijerink et al., "Novel ring resonator-based integrated photonic beamformer for broadband phased array receive antennas-part II: experimental prototype," Journal of Lightwave Technology, vol. 28, no. 1, pp. 19-31, 2010. 
[10] N. A. Riza, "An acoustooptic-phased-array antenna beamformer for multiple simultaneous beam generation," IEEE Photonics Technology Letters, vol. 4, no. 7, pp. 807-809, 1992.

[11] R. D. Esman, M. Y. Frankel, J. L. Dexter et al., "Fiber-optic prism true time-delay antenna feed," IEEE Photonics Technology Letters, vol. 5, no. 11, pp. 1347-1349, 1993.

[12] B. Vidal, M. A. Piqueras, and J. Martí, "Multibeam photonic beamformer based on optical filters," IEE Electronics Letters, vol. 42, no. 17, pp. 980-981, 2006.

[13] L. Yaron, R. Rotman, S. Zach, and M. Tur, "Photonic beamformer receiver with multiple beam capabilities," IEEE Photonics Technology Letters, vol. 22, no. 23, pp. 1723-1725, 2010.

[14] B. R. Elbert, The Satellite Communications Applications Handbook, Artech House, Norwood, NJ, USA, 2004. 

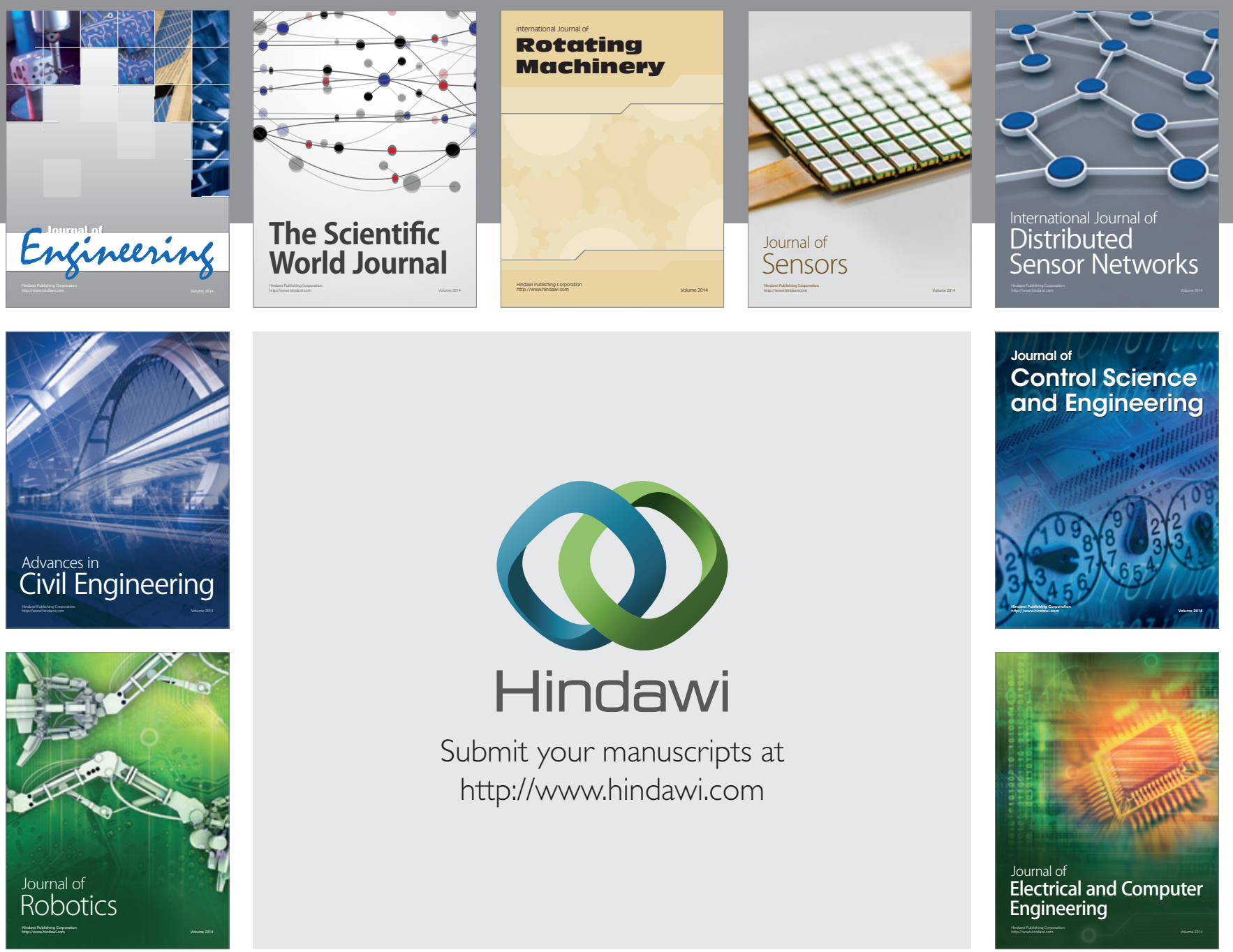

Submit your manuscripts at

http://www.hindawi.com
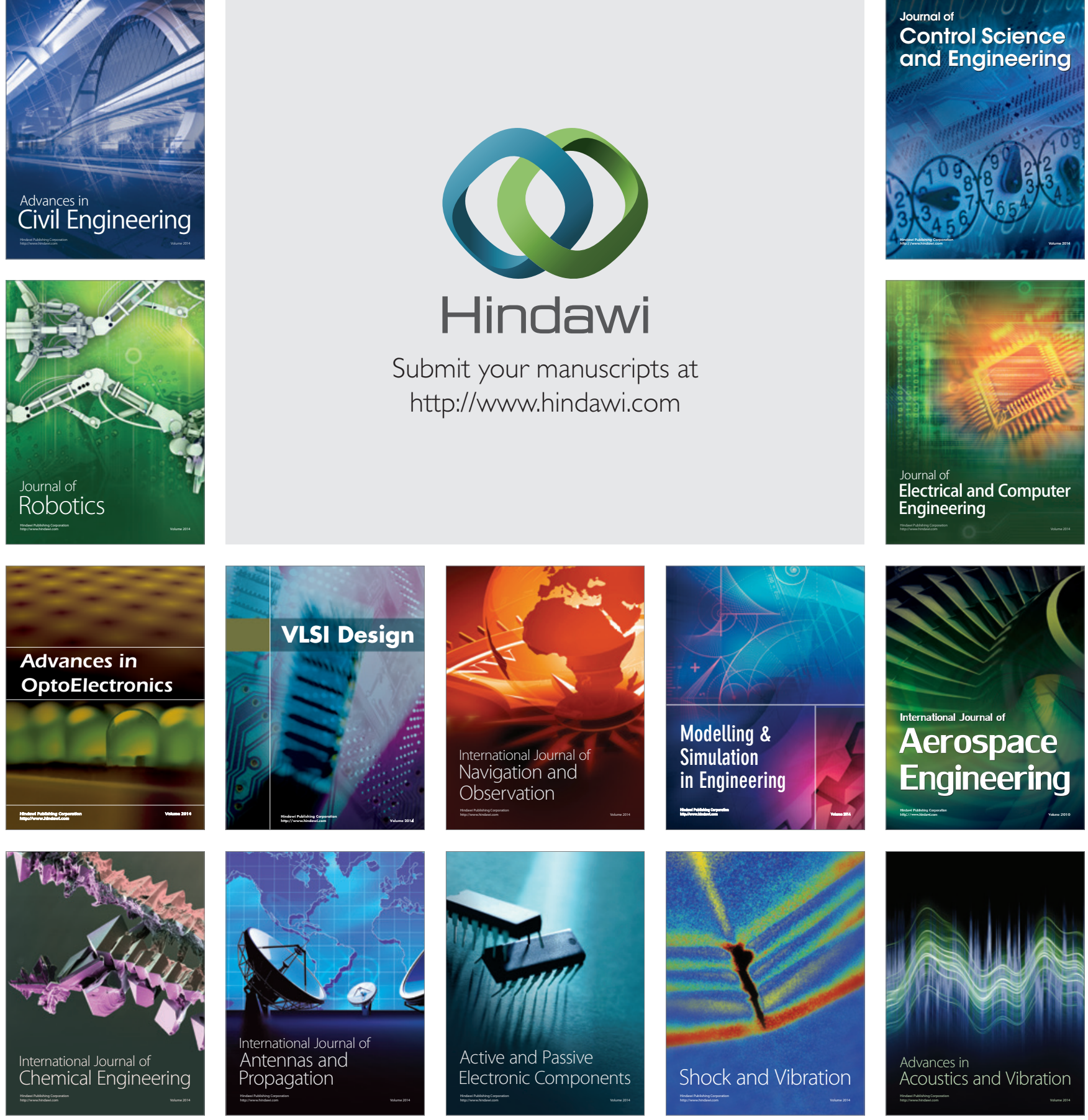